\title{
Fridolin Schuler - ein (vergessener) sozialmedizinischer Pionier
}

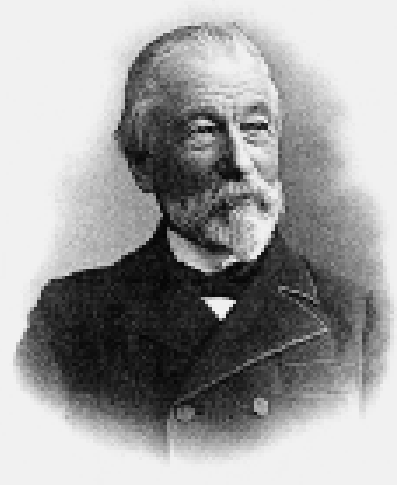

Constantin Schuler

* Mitteilung der Gemeinde Mollis: Schuler starb am 8. Mai 1903 in Aarburg. Sein Grabmal auf dem heimatlichen Friedhof wird von der Gemeinde in ehrender Weise dauernd erhalten. Am 19. Juli 1946 brachte das eidgenössische Fabrikinspektorat an Schulers Wohnhaus im Hertenacker eine steinerne Gedenktafel an mit der Inschrift:

In diesem Hause lebte 1864 bis 1903 Dr. med. Fridolin Schuler, sozialpolitischer Förderer der Arbeiterschutzgesetzgebung, glarnerischer und eidgenössischer Fabrikinspektor, Freund des Volkes.

Korrespondenz:

Dr. med. Constantin Schuler Staag

CH-9402 Mörschwil

con-son@gmx.ch
Im letzten Drittel des 19. Jahrhunderts begann man sich in Deutschland unter Otto Graf Bismarck ernsthaft mit der Einführung eines allgemeinen Versicherungsschutzes gegen Krankheit, Unfall und Altersgebrechen für sozial Schwache - man dachte an 10\% der Bevölkerung - zu befassen. Die «Kaiserliche Botschaft» von 1881 hat damals als «Magna Charta» die Welt erstaunt und vielerorts eine Sozialgesetzgebung in Gang gebracht.

In der Schweiz war die treibende Kraft ein Landarzt im glarnerischen Mollis, Dr. Fridolin Schuler, seit 1867 nebenamtlich Fabrikinspektor des Kantons Glarus. Als unermüdlicher Vorkämpfer für bessere hygienische Verhältnisse am Arbeitsplatz veröffentlichte er seit 1872 laufend Publikationen $\mathrm{zu}$ arbeitsmedizinischen Themen. So 1875 über die «Glarner Baumwollindustrie und ihr Einfluss auf die Gesundheit der Arbeiter» und 1876 über die gesammelten «Glarner Totenscheine, vornehmlich zum Zwecke nachzuweisen, welche Erkrankungsformen bei dieser oder jener Berufsgruppe am häufigsten zum Tode führen». 1883 schrieb er über die «Gefährliche Bearbeitung des gelben Phosphors in den Zündholzfabriken» und 1899 «Die soz. Zustände in der Seidenindustrie der Ostschweiz». Das Verzeichnis seiner Arbeiten ist beeindruckend lang und umfasst gegen 80 Publikationen. Daneben hielt er laufend Vorträge, so über die Begräbnisordnung, die Schulgesundheitspflege, das Impfwesen und ab 1873 über die Notwendigkeit der Gründung eines Kantonsspitals Glarus.

Als der Landarzt in der Glarner MedizinischChirurgischen Gesellschaft immer wieder auf die unverantwortlichen hygienischen Zustände in den Fabriken, den Zwölfstundentag und die Kinderarbeit zu sprechen kam, spotteten seine Kollegen: «Schuster, bleib bei Deinem Leisten! Ein Arzt hat die Menschen bei Geburt, Krankheit und Tod zu begleiten und damit basta!» Schuler liess sich aber nicht beirren, was ihm mit der Zeit grimmige Feindschaften einbrachte.

Im Oktober 1877 wurde nach einem leidenschaftlich geführten Abstimmungskampf das Eidgenössische Fabrikgesetz knapp angenommen und Schuler zum ersten Fabrikinspektor der Schweiz bestimmt. In einer Orientierungsreise durch das Land besuchte er im Auftrage des Bundesrates in den folgenden neun Monaten 268 «Etablissements», die 105 verschiedenen Industrien angehörten. 1879 lieferte er dem mit ihm befreundeten Glarner Bundesrat Joachim Heer einen detaillierten Bericht ab und formulierte mehrere Anträge. Insbesondere schlug er eine Ergänzung der Fabrikliste vor, «da sich allerlei Industrien dem Fabrikgesetz entziehen wollten». In der Folge wurde jegliche Kinderarbeit unter 14 Jahren verboten und bald der Elfstundentag eingeführt. Offenbar, wie der Autor schreibt, weil sich jetzt «die Ärzte überhaupt um das Zustandekommen des Fabrikgesetzes viel Mühen gegeben hatten».

Zu seinem 70. Geburtstag am 1. April 1902 erhielt Schuler im Auftrag des Bundesrates einen vergoldeten Becher von prachtvoll getriebener Art mit sehr anerkennender Widmung und in der Aula der Universität Zürich, von seinem Freund Prof. O. Roth überreicht, den Ehrendoktor der Staatswissenschaftlichen Fakultät.

Ein Jahr später, an seinem Todestag* am 8. Mai 1903, lag das Manuskript der «Erinnerungen eines Siebenzigjährigen» druckfertig vor. Zwei Monate danach unterzeichnete Dr. Johannes Seitz, ein Zürcher Arzt und langjähriger Freund des Verstorbenen, als Bevollmächtigter der Witwe, Frau Emma Schuler-Kubly, einen Verlagsvertrag mit Huber \& Co. in Frauenfeld. Darin heisst es: Für Drucklegung und Herausgabe der als Selbstbiographie betitelten «Erinnerungen eines Siebenzigjährigen» beträgt der Preis für die 1. Auflage mit 2000 Exemplaren Fr. 500.- plus 25 Rappen für jedes verkaufte Buch. Über eine 2. Auflage ist nichts bekannt.

\section{Erinnerungen eines Siebenzigjährigen}

Auf 160 Seiten berichtet der Landarzt und spätere Eidgenössische Fabrikinspektor in den «Erinnerungen eines Siebenzigjährigen» autobiographisch über sein bewegtes Leben, angefangen von der Geburt am 1. April 1832 und seiner unbe- 
schwerten Jugend im elterlichen Pfarrhaus zu Bilten. Dann von den Erlebnissen als Gymnasiast an der «von den Glarnern hochgeschätzten Bezirksschule» in Aarau. Mit der anschliessenden Schilderung der Studentenzeit gewährt uns der Autor tiefe Einblicke in die bewegten «Wanderjahre» der angehenden Mediziner von damals, als sie durch halb Europa von einer Universität zur anderen zogen, um möglichst viele berühmte Autoritäten zu hören.

Schuler begann 1851 an der Universität Zürich sein Medizinstudium mit Anatomie beim bekannten Physiologen C. Ludwig, wechselte nach 2 Jahren zu Professor R. Virchow in Würzburg und später nach Wien, wo ihm die noch auf lateinisch gehaltene Vorlesung von C. Oppolzer über «Sorgfältige Differentialdiagnose» und das «ungeheure Material» der Internen Klinik von Hebra imponierten. Anschliessend folgte ein Aufenthalt beim berühmten Geburtshelfer Chiari im «tausendtürmigen Prag» und als Abschluss ein solcher beim Chirurgen Nélaton in Paris.

In die Heimat zurückgekehrt legte Schuler erfolgreich seine Examen für die kantonale Promovierung zum Arzt ab - eine eidgenössische gab es damals noch nicht. 1855 wagte er den Schritt in die Praxis und mit Emma Kubly in den Ehestand. Die Tätigkeit als Dorfarzt in Mollis und im bergigen Glarnerland beschreibt er als sehr befriedigend, aber körperlich ausserordentlich anstrengend. So war er froh, die Praxis nach 22 Jahren einem Neffen übergeben zu können. Um so mehr, als er sich in den letzten Jahren ohnehin immer stärker mit öffentlicher Gesundheitspflege und Gewerbehygiene befasst hatte. Auch war er mit der herrschenden Gesundheitspolitik nicht einverstanden, insbesondere der «Freigebung der Praxis» durch die Landsgemeinde am 3. Mai 1874 an «zweifelhafte Existenzen und den letzten Quacksalber».

Und so trat er 1878 mit Begeisterung auf das Angebot ein, die neugeschaffene Stelle als Eidgenössischer Fabrikinspektor zu übernehmen. Ausführlich und spannend schildert Schuler auf 80 Seiten seine mannigfachen Tätigkeiten als beamteter Arzt im öffentlichen Gesundheitsdienst verbunden mit zahlreichen sozialpolitischen Vorstössen auf kantonaler und auf Bundesebene. So auch die Teilnahme am Internationalen Unfallkongress 1891 in Bern, wo er als Delegierter des Bundesrates einen Vortrag über «Krankheit-, Unfall- und Invalidenversicherung und ihre Verbindung» hielt. Dank seiner zahlreichen Veröffentlichungen auf gewerbehygienischem und sozialpolitischem Gebiet kamen «sehr oft Besucher aus aller Herren Länder, um unsere schweizerischen Einrichtungen kennenzulernen. Viele bekannte Ärzte, Professoren und andere einflussreiche Leute, nicht nur aus Skandinavien, wollten unsere Meinung zu entsprechenden Plänen in ihren Heimatländern hören.»

Man ist heute davon beeindruckt, was von ein paar unentwegten Pionieren in der zweiten Hälfte des 19. Jahrhunderts gegen grosse Widerstände und bittere Gegnerschaft erstritten und erträumt wurde und vielerorts recht bald in die Gesetzgebung eingeflossen ist. Wenige haben damals, nach Schuler «umgeben von vielen Feinden», die in einem Jahrhundert wohl grösste sozialpolitische Umwälzung in der bekannten Menschheitsgeschichte in Gang gebracht.

Mit Genugtuung betrachtet der Autor in besinnlichem Rückblick die während seines Lebens eingetretenen medizinischen und sozialen Fortschritte. Und er bewertet die Verhältnisse hierzulande, verglichen mit dem, was er vielfach im Ausland gesehen hat, als erfreulich positiv. So schreibt er namentlich: «Weil hier Millionärsund Bettlersohn die gleiche Schule aufnimmt, oft ein einfacher Bauer oder Fabrikarbeiter die höchste Stellung in seinem fabrikreichen Dorfe einnimmt. wo alle Stände sich mischen und jeder mit der Denk-, Sprach- und Lebensweise des andern vertraut wird. Wie sehr mildert dies bei uns die Gegensätze, die ja freilich in manchen Dingen doch noch schroff genug zu Tage treten.»

Der Verlag von Huber \& Co. in Frauenfeld hat die «Erinnerungen eines Siebenzigjährigen» mit einem Porträt Fridolin Schulers und einem Verzeichnis seiner über 80 Arbeiten versehen im

Jahre 1903 in Buchform herausgebracht.**

\footnotetext{
Hilfe und Einsicht in die hundert jährigen Verlagsakten herzlich gedankt.

Dem Staatsarchiv des Kantons Thurgau sei für die freundliche
} 